\title{
openheart Endovascular management of renal artery aneurysms using the multilayer flow modulator
}

\author{
Sherif Sultan, ${ }^{1,2}$ Mahmoud Basuoniy Alawy, ${ }^{1}$ Rita Flaherty, ${ }^{1}$ Edel P Kavanagh, ${ }^{2}$ \\ Mohamed Elsherif, ${ }^{1}$ Ala Elhelali, ${ }^{1}$ Florian Stefanov, ${ }^{2}$ Violet Lundon, ${ }^{1}$ Niamh Hynes ${ }^{2}$
}

To cite: Sultan S, Basuoniy Alawy M, Flaherty R, et al. Endovascular management of renal artery aneurysms using the multilayer flow modulator. Open Heart 2016:3:e000320. doi:10.1136/openhrt-2015000320

Received 4 August 2015 Revised 10 December 2015 Accepted 12 January 2016

\section{(a) CrossMark}

${ }^{1}$ Department of Vascular and Endovascular Surgery, Western Vascular Institute, Galway University Hospital, National university of Ireland, Galway, Ireland

${ }^{2}$ Department of Vascular and Endovascular Surgery, Galway Clinic, Galway, Ireland

Correspondence to Prof Sherif Sultan; sherif.sultan@hse.ie

\section{ABSTRACT}

Objective: Our aim was to describe our experience of the Multilayer Flow Modulator (MFM, Cardiatis, Isnes, Belgium) used in the treatment of type III renal artery aneurysms (RAA).

Methods: This is a single-centre study. 3 patients ( 2 men and 1 woman; mean age 59 years; range 41-77 years) underwent treatment of a type III renal artery aneurysm using the MFM. The indications were a $23.9 \mathrm{~mm}$ type III RAA at the bifurcation of the upper and lower pole vessels, with 4 side branches; a $42.4 \mathrm{~mm}$ type III saccular RAA at the renal hilum; and a $23 \mathrm{~mm}$ type III RAA at the origin of the artery, supplying the upper pole.

Results: Patients had a mean follow-up of 27 months, and were assessed by perioperative renal function tests, and repeat postoperative CT scan. There were no immediate postoperative complications or mortality. The first patient's aneurysm shrank by $8.6 \mathrm{~mm}$, from 23.9 to $15.3 \mathrm{~mm}$ over 19 months, with all 4 side branches remaining patent. The largest aneurysm at $42.4 \mathrm{~mm}$ completely thrombosed, while the renal artery remained patent to the kidney. The final patient refused to have any follow-up scans but had no deterioration in renal function below $30 \mathrm{~mL} / \mathrm{min}$, and no further symptoms reported

Conclusions: The MFM is safe and effective in the management of patients with complex renal artery aneurysms. The MFM can be used to treat branched or distal renal artery aneurysms with exclusion of the aneurysm from the circulation, while successfully preserving the flow to the side branches and kidney. Initial results are promising, however, longer follow-up and a larger cohort are required to prove the effectiveness of this emerging technology.

\section{INTRODUCTION}

Renal artery aneurysms (RAA) are a rare but serious pathology. They are found in $0.7 \%$ of autopsies and up to $1 \%$ of angiographic investigations. ${ }^{1}$ They can lead to renovascular hypertension, thromboembolic events and rupture. Rupture of RAA is associated with a significant mortality or loss of a kidney, especially during pregnancy. ${ }^{2}$ RAA are classified

\section{KEY QUESTIONS}

What is already known about this subject?

- The Multilayer Flow Modulator (MFM, Cardiatis, Isnes, Belgium) has been used to treat peripheral, visceral and aortic aneurysms. Previous studies show that it has also been used with success to treat renal artery aneurysms. The MFM design allows blood to perfuse through the mesh in a manner that maintains collateral branch patency, while modulating the flow from turbulent to laminar within the device and the aneurysm sac. Laminar flow in the aneurysm encourages the formation of organised thrombus, thereby stabilising aneurysm size and reducing risk of rupture.

What does this study add?

- This study outlines our own centre's mid-term experience with treating complex renal artery aneurysms. These patients would otherwise have been treated via open surgery. The results presented affirm those already available, showing successful intervention with the MFM. It encourages aneurysm sac shrinkage, while maintaining collateral branch patency.

How might this impact on clinical practice?

- Currently, the treatment of renal artery aneurysms includes renal artery reconstruction, with or without bypass, with an autologous conduit, aneurysmectomy and endovascular treatment with stenting and catheter-directed embolisation. Endovascular treatment can now give patients an option with reduced perioperative time, hospital stay, mortality and morbidities that are associated with major abdominal surgery.

according to the angiographic classification system. ${ }^{3}$ Type I RAA are saccular and arise from either the main renal artery trunk, or proximally from a large segmental artery; type II are a fusiform shape and occur at the main renal artery or proximal segmental artery; type III aneurysms are more distal, affecting the small segmental or intraparenchymal artery. Associated diseases of RAA 
include hypertension $(73 \%)$, renal artery fibrodysplasia $(34 \%)$, systemic atherosclerosis $(25 \%)$, and extrarenal aneurysms $(6.5 \%)$. There is twice the incidence in women than men, ${ }^{4}$ with $55 \%$ of RAA being asymptomatic. ${ }^{4}$ It is generally accepted that aneurysms of $20 \mathrm{~mm}$ or greater in diameter should be treated. ${ }^{5}$ Moreover, those of childbearing age or antenatal with evidence of emboli, and symptomatic patients (with pain, hypertension or haematuria), or rapidly expanding aneurysms should be treated.

An alternative modality of treatment of RAA is the Multilayer Flow Modulator (MFM; Cardiatis, Isnes, Belgium; figure 1), which disagrees in terms of concept with conventional treatment modes. The MFM is currently widely used in aortic, ${ }^{6}$ visceral, ${ }^{7}$ and peripheral aneurysm repair, ${ }^{8}$ and aortic dissection. ${ }^{9}$ Previous studies by Polydorou et $a l^{8}{ }^{8}$ Henry et $a l^{10}{ }^{11}$ Meyer $e t a l,{ }^{12}$ Flis et $a l^{13}$ and Ruffino and Rabbia ${ }^{14}$ show it has also been used with success to treat renal artery aneurysms. It harnesses the body's innate physiological processes to modulate the aneurysm with no risk of critical shuttering or loss of native side branches. The MFM also offers less operative trauma, shorter procedure time and reduced hospital stay. It's simplicity, consistency and reproducibility of results attracted enthusiasm among interventionists. Our aim was to describe our mid-term experience of the MFM stent, used in the treatment of

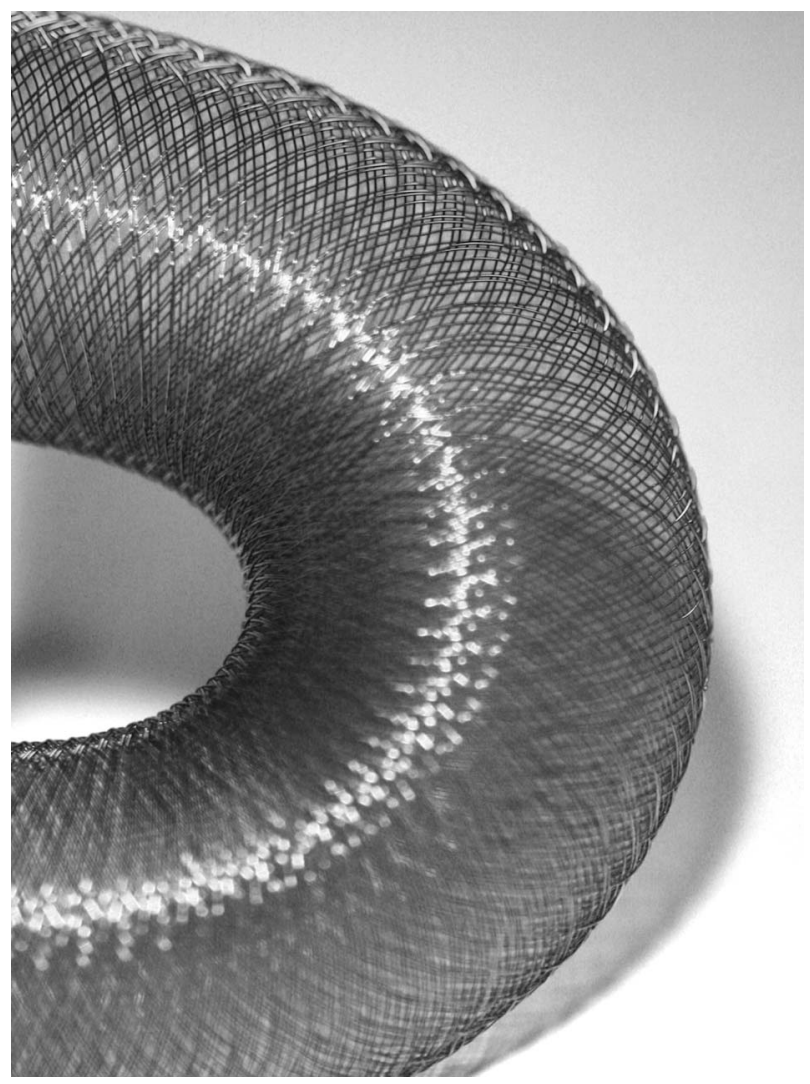

Figure 1 The Multilayer Flow Modulator (Cardiatis, Isnes, Belgium). complex RAA, assessing patency of side branches and aneurysm shrinkage.

\section{METHODS}

Three patients (2 men and 1 woman) with a mean age of 59 years and range 41-77 years underwent RAA repair with the MFM. Preoperative work-up consisted of a contrast-enhanced CT scan with $1 \mathrm{~mm}$ axial slices. All patients were symptomatic and presented with constant pain in the right side of the abdomen or loin. One patient had a history of hypertension, and the CT scan showed bilateral renal cysts. The indications were a $23.9 \mathrm{~mm}$ type III right renal aneurysm at the bifurcation of the upper and lower pole vessels with four side branches (figure 2); a $42.4 \mathrm{~mm}$ type III right renal artery saccular aneurysm (figure 3 ); and a $23 \mathrm{~mm}$ type III right renal artery aneurysm coming off the upper pole artery (figure 4 ).

The diameters and lengths of each aneurysm and the arterial landing zones were measured via CT scan. A $6 \times 60 \mathrm{~mm}$ (diameter $\times$ length), $8 \times 50 \mathrm{~mm}$ and $6 \times 30 \mathrm{~mm}$ MFM devices were selected. MFM intervention was performed under endotracheal general anaesthesia. An intraoperative loading dose of 4000 IU heparin was given intravenously to the patient. Duplex-guided percutaneous cannulation of the right common femoral artery with a $5 \mathrm{~F}$ sheath was then carried out. The

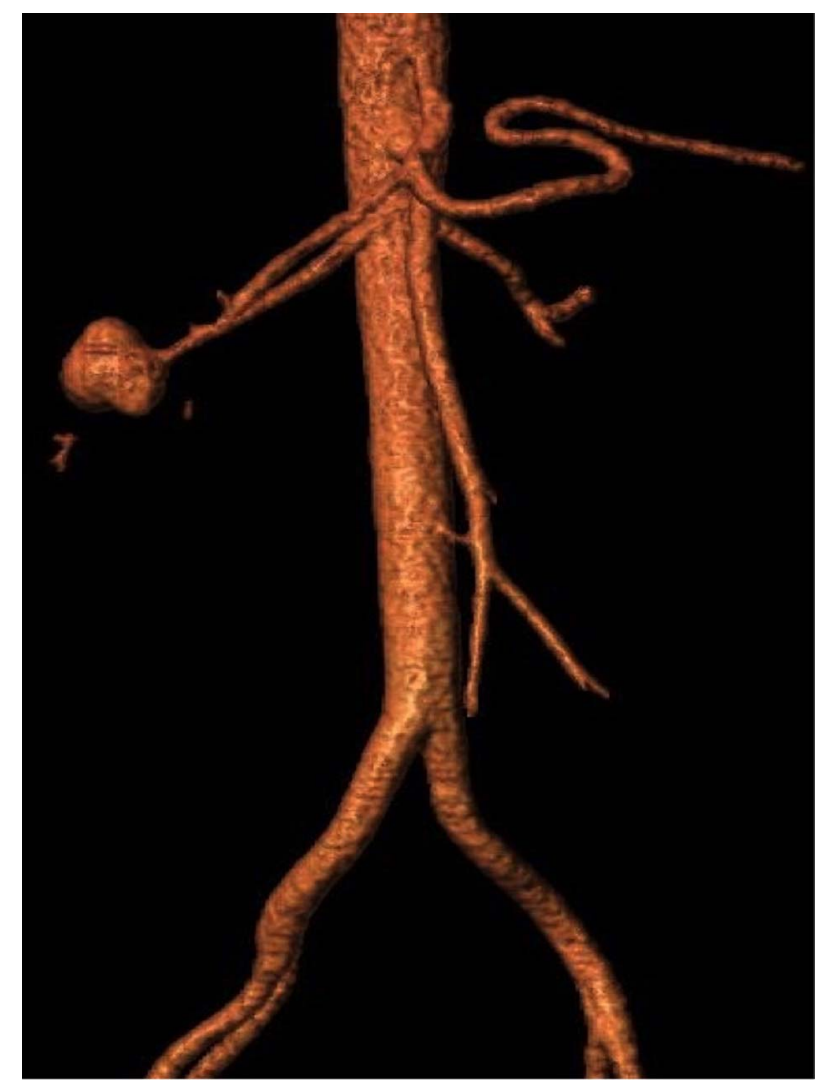

Figure 2 Three-dimensional reconstruction of a type III right renal artery aneurysm (case 1). 


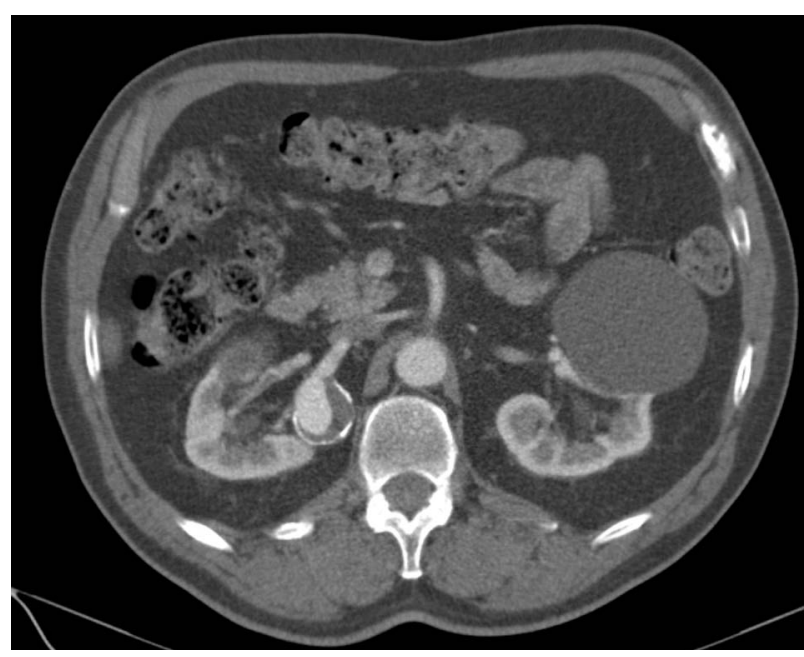

Figure 3 CT angiography showing a type III saccular right renal artery aneurysm, with decreased cortex to medulla parenchyma ratio (case 2).

Terumo guide wire was advanced into the aorta and then replaced with a 7F destination sheath. The renal vessel was cannulated with a paediatric selective visceral catheter. The $7 \mathrm{~F}$ sheath was then advanced inside the renal artery. The MFM was deployed in the target vessel with oversizing by $15 \%$, and landed distally in the largest runoff side branch. Once the procedure was complete, the guide wires were withdrawn and closure of the right femoral puncture was carried out with a Perclose device (Abbott Laboratories, Illinois, USA). Preoperative and postoperative estimated glomerular filtration rate (eGFR) to assess renal function during the procedure was also measured, defined as severe kidney damage below $30 \mathrm{~mL} / \mathrm{min}$. Primary technical success was defined as no immediate failures and no postoperative complications, including stent thrombosis, migration and infection. Primary clinical success was defined as

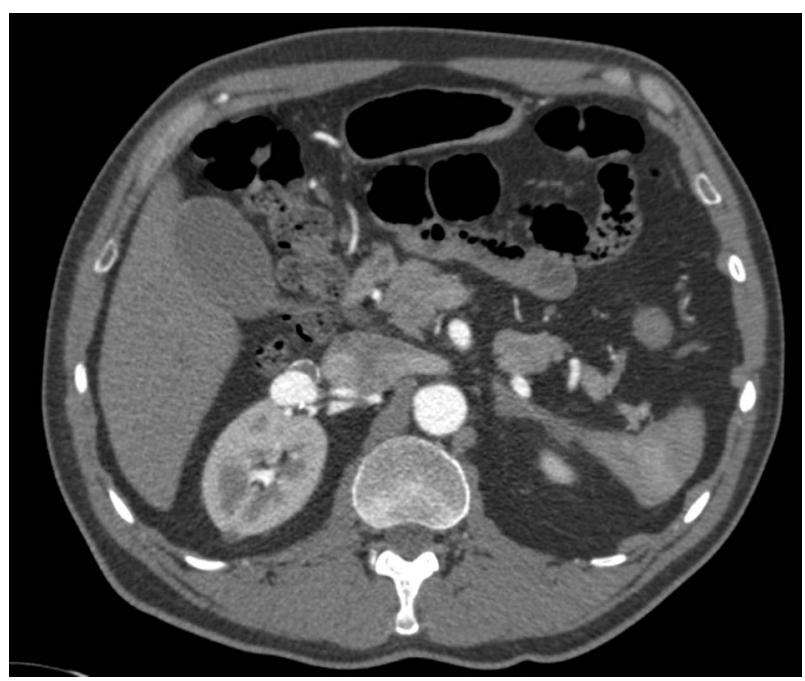

Figure 4 CT angiography showing an upper pole type III right renal artery aneurysm (case 3 ). freedom of mortality due to aneurysm rupture, and shrinkage or stabilisation of the aneurysm sac.

\section{RESULTS}

Postoperative follow-up with CT angiography was conducted at 6 and 19 months for case 1, and 35 and 47 months for case 2. One patient (case 3) refused further follow-up CT scans. Mean follow-up was 27 months. Case 2 was carried out in 2009, while cases 1 and 3 were carried out 2011. Primary technical success was $100 \%$. There were no immediate failures and no postoperative complications including stent thrombosis, migration and infection.

Case 1 showed a normal preoperative eGFR of $>90 \mathrm{~mL} /$ min, which remained unchanged postoperatively. Final follow-up CT scan (19 months) showed the aneurysm shrunk by $36 \%$ (from 23.9 to $15.3 \mathrm{~mm}$ ) with all four side
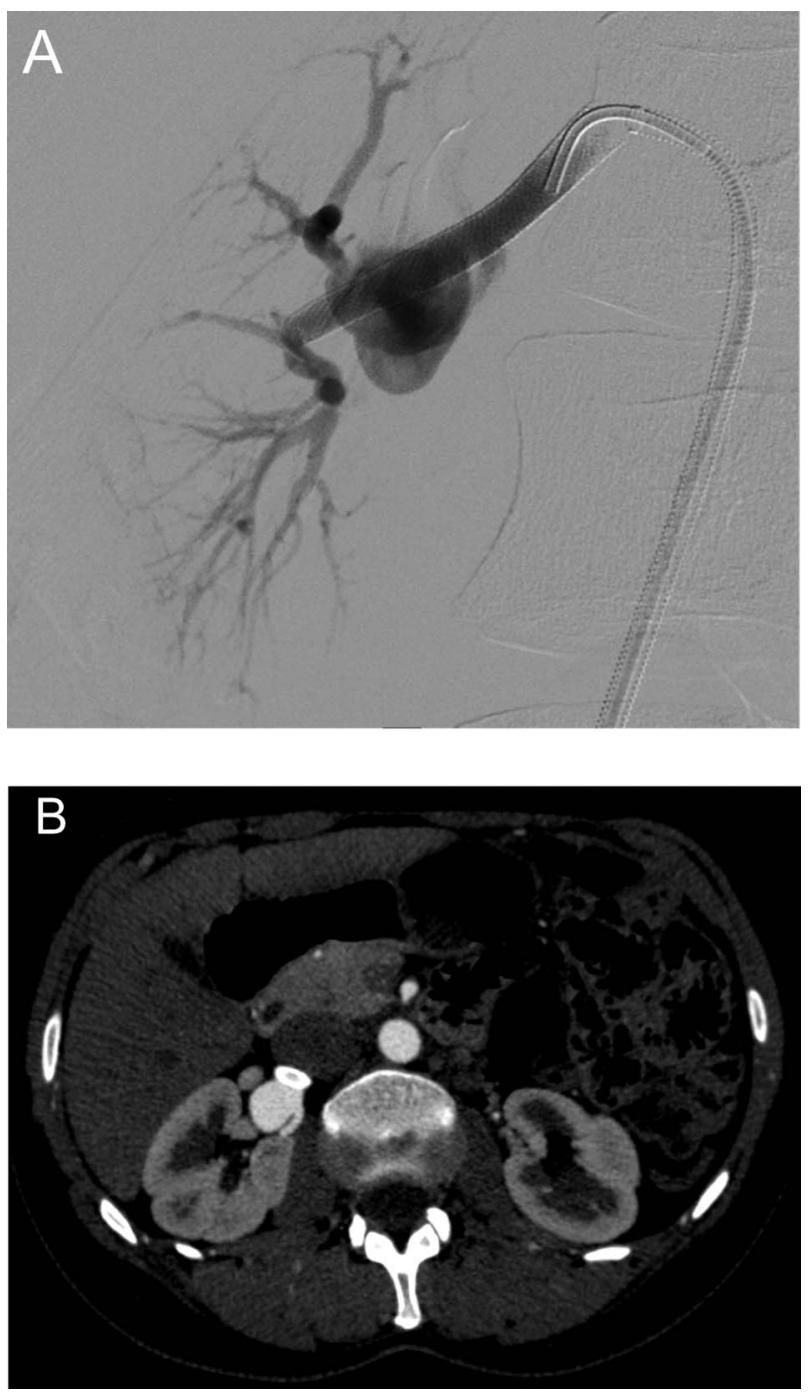

Figure 5 (A) Digital subtraction angiogram during postdeployment of the Multilayer Flow Modulator (MFM) in the right renal artery, demonstrating patency of all side branches (case 1). (B) Follow-up (19 months) CT angiography showing aneurysm side branch patency (case 1). 


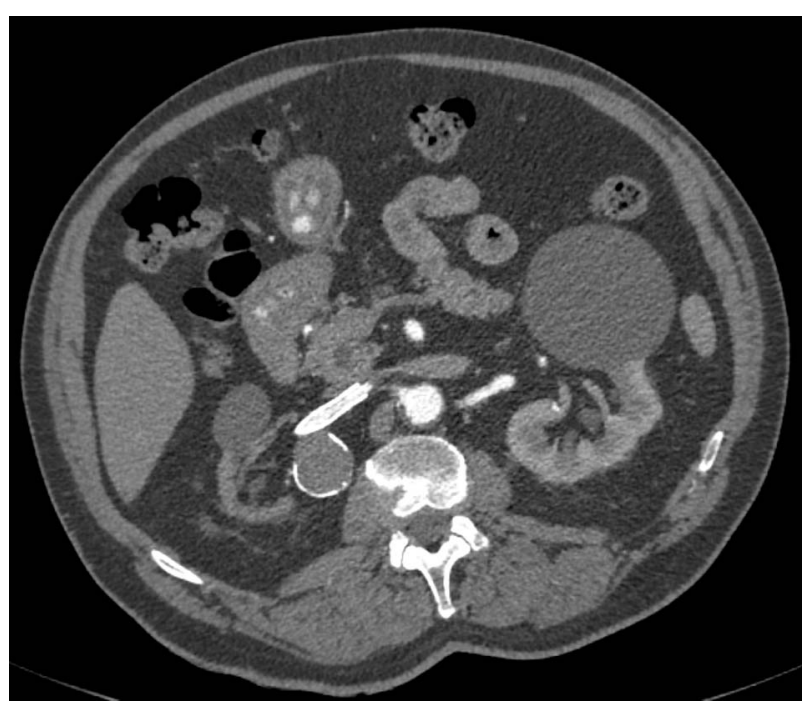

Figure 6 Follow-up (47 months) CT angiography of a type III saccular right renal artery aneurysm (RAA) showing no contrast agent entering the aneurysm sac with sac thrombosis (case 2).

branches remaining patent postoperatively (figure 5). Case 2 recorded a preoperative eGFR of $69 \mathrm{~mL} / \mathrm{min}$, which declined postoperatively to $55 \mathrm{~mL} / \mathrm{min}$. The aneurysm had completely thrombosed in a final follow-up CT scan (47 months; figure 6). Aneurysm shrinkage of $9.4 \%$ (from 42.4 to $38.4 \mathrm{~mm}$ ) was measured. Case 3 had a preoperative eGFR of $>90 \mathrm{~mL} / \mathrm{min}$, which declined postoperatively to $74 \mathrm{~mL} / \mathrm{min}$. A perioperative CT scan was carried out showing the deployed MFM (figure 7). This patient refused follow-up CT scans, however, it was noted that this patient did not experience any severe deterioration in renal function or further symptoms.

\section{DISCUSSION}

Treatment options for RAA include renal artery reconstruction, with or without bypass, with an autologous conduit, aneurysmectomy, and endovascular treatment

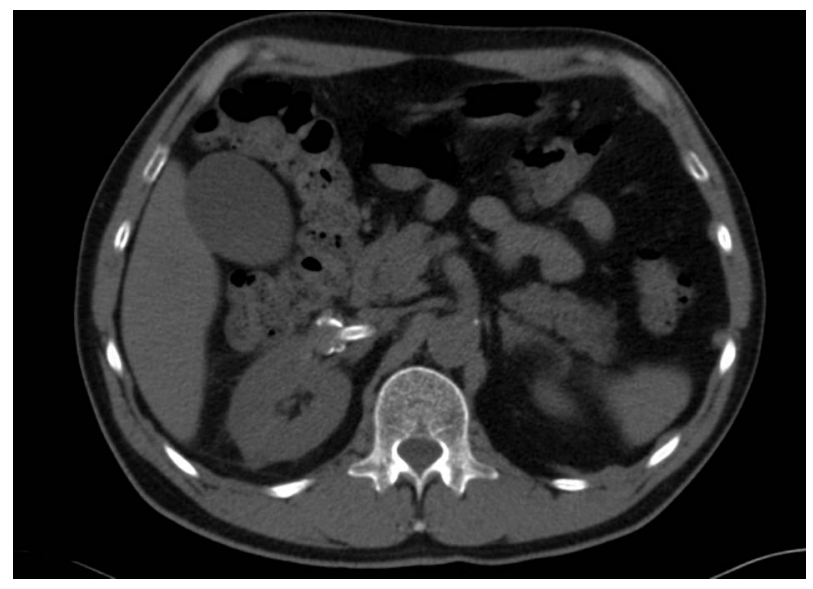

Figure 7 Perioperative (4 days) CT angiography of a type III right renal artery aneurysm (RAA), showing the deployed Multilayer Flow Modulator (MFM) (case 3). with stenting and catheter-directed embolisation. Open surgery to treat these aneurysms involves major abdominal surgery with its concomitant mortalities and morbidities, such as unplanned nephrectomy or dialysisdependant renal failure. In a case series of a high volume centre in 96 operated patients, eight patients had unplanned nephrectomies due to technical complications. ${ }^{4}$ An endovascular approach is becoming an increasingly popular route of repair, however, if an RAA has single or multiple arterial branches exiting directly from the aneurysm sac, endovascular methods of treatment may result in obstruction of the branch or branches, reducing perfusion to the kidney. In complex hilar aneurysm cases involving multiple arterial branches, extracorporeal vascular reconstruction with auto-transplantation is often the only feasible option of repair. The MFM, therefore, provides a viable new off-the-shelf endovascular treatment option for RAA.

With a porosity of approximately $65-75 \%$, the MFM design allows blood to perfuse through the mesh in a manner that maintains collateral branch patency, while modulating the flow from turbulent to laminar within the device and the aneurysm sac. Laminar flow in the aneurysm encourages the formation of organised thrombus, thereby stabilising aneurysm size and reducing risk of rupture. The MFM has been used successfully to treat peripheral and visceral artery aneurysms, ${ }^{7} 8$ 10-16 complex thoracoabdominal aneurysms, and aortic type B dissections, ${ }^{6} \quad 9$ 17-19 These studies reported successful treatment of the aneurysms by reduction in flow velocity within the aneurysm sac and surrounding branches, leading to the formation of an organised thrombus.

The introduction of the MFM to the mainstream vascular practice has forced us to re-evaluate our core concepts of aneurysm management and add the fourth dimension of time for the conclusion of a clinical outcome, moreover, it shifted our focus from anatomical to physiological therapies. The key components of MFM function are its manipulation of blood flow, preservation and enhancement of flow into arterial side branches, compliance, encouragement of endothelialisation and influence on thrombus formation, all of which reduce peak wall stresses while simultaneously enhancing wall strength and promoting healing. ${ }^{20} 21$

Type III interlobar and/or intraparenchymal RAA, have previously been managed by super-selective embolisation techniques, in order to limit the number of vessels being occluded. This results in thrombosis of the end arteries and renal infarction. Moreover, there is an increase in hypertension, and non-target embolisation with migration of coils into the main renal artery and systemic circulation. ${ }^{3}$ 22-29 Type III intrahilar RAA require adequate distal vascular control for reconstruction, and an ex vivo approach is always recommended. ${ }^{30}$ This major surgery can only be done in high deliberate practice volume centres, but patients' demand for less invasive techniques allow us to use the MFM. 
There have been complications reported in relation to the MFM such as stent foreshortening and dislocation. ${ }^{31-33}$ These types of complications are often related to the fact that this technology was globally disseminated prior to controlled clinical trials and was indiscriminately used by eager operators who experimented with the device. This resulted in dismal clinical outcomes. Cases in which the MFM is contraindicated have been reported previously. ${ }^{34}$

From our experience, treatment of RAA with the MFM is a promising avenue. Primary technical success was $100 \%$. There were no immediate failures and no postoperative complications, including stent thrombosis, migration and infection. All operations were performed via a percutaneous femoral approach with all stents being deployed without any complication. One case showed complete thrombosis of the aneurysm sac at follow-up 47 months postoperatively. The four collateral branches arising from the aneurysm in one patient remained patent 19 months postoperatively, and there was no significant compromise to renal function subsequent to the implantation. Postoperative outcomes in the literature showed that the MFM proved a safe technique during perioperative hospital stays, with no reported deaths, paraplegia, cerebrovascular accident, visceral or renal compromise. ${ }^{9-12} 20-22$

\section{CONCLUSION}

On the basis of our clinical experience, treatment of renal artery aneurysms with the MFM appears to be a safe viable alternative to other current surgical treatments. The MFM has shown its ability to treat branched or distal renal artery aneurysms with exclusion of the aneurysm from the circulation, while successfully preserving the flow to the side branches and kidney, avoiding autotransplantation of the kidney. Sac shrinkage occurred over the duration of the follow-up with no complications, such as branch occlusion, device migration or thrombosis. A larger cohort of patients, with longer follow-up is required to prove the efficacy of this emerging disruptive technology.

Contributors SS designed the whole study and is the guarantor. SS, MBA, RF, EPK, ME and VL collected the data. SS, EPK, RF, ME, MBA, NH, FS, VL and $A E$ drafted the manuscript.

Competing interests None declared.

Provenance and peer review Not commissioned; externally peer reviewed.

Data sharing statement No additional data are available.

Open Access This is an Open Access article distributed in accordance with the Creative Commons Attribution Non Commercial (CC BY-NC 4.0) license, which permits others to distribute, remix, adapt, build upon this work noncommercially, and license their derivative works on different terms, provided the original work is properly cited and the use is non-commercial. See: http:// creativecommons.org/licenses/by-nc/4.0/

\section{REFERENCES}

1. Tham G, Ekelund L, Herrlin K, et al. Renal artery aneurysms: natural history and prognosis. Ann Surg 1983;197:348-52.

2. Cohen JR, Shamash FS. Ruptured renal artery aneurysm during pregnancy. J Vasc Surg 1987;6:51-9.

3. Rundback JH, Rizvi A, Rozenblit GN, et al. Percutaneous stent-graft management of renal artery aneurysms. $J$ Vasc Interv Radiol 2000;11:1189-93.

4. Henke PK, Cardneua JD, Welling TH, et al. Renal artery aneurysms: a 35 year clinical experience with 252 aneurysms in 168 patients. Ann Surg 2001;234:454-62; discussion 462-3.

5. Eskandari MK, Resnick SA, Aneurysms of the renal artery. Semin Vasc Surg 2005;18: 202-8.

6. Vaislic CD, Fabiani JN, Chocron S, et al. One-year outcomes following repair of thoracoabdominal aneurysms with the Multilayer Flow Modulator: report from the STRATO trial. J Endovasc Ther 2014;21:85-95.

7. Balderi A, Antonietti A, Pedrazzini F, et al. Treatment of visceral aneurysm using multilayer stent: two-year follow-up results in five consecutive patients. Cardiovasc Intervent Radiol 2013;36:1256-61

8. Polydorou A, Henry M, Bellenis I, et al. Endovascular treatment of arterial aneurysms with side branches-a simple method. Myth or reality? Hosp Chron 2010;5:88-94.

9. Chocron S, Vaislic C, Kaili D, et al. Multilayer stents in the treatment of thoraco-abdominal residual type B dissection. Interact Cardiovasc Thorac Surg 2011;12:1057-9.

10. Henry M, Polydorou A, Frid N, et al. Treatment of renal artery aneurysm with the multilayer stent. J Endovasc Ther 2008;15:231-6.

11. Henry M, Benjelloun A, Henry I, et al. The multilayer flow modulator stent for the treatment of arterial aneurysms. J Cardiovasc Surg (Torino) 2013;54:763-83.

12. Meyer C, Verrel F, Weyer G, et al. Endovascular management of complex renal artery aneurysms using the multilayer stent. Cardiovasc Intervent Radiol 2011;34:637-41.

13. Flis V, Matela J, Breznik S, et al. Endovascular repair of renal artery aneurysm with the multilayer stent-a short report. Slovenian Med J 2012;81:753-8.

14. Ruffino MA, Rabbia C. Endovascular repair of peripheral and visceral aneurysms with the cardiatis Multilayer Flow Modulator: one-year results from the Italian multicenter registry. $J$ Endovasc Ther 2012;19:599-610.

15. Ferrero E, Ferri M, Viazzo A, et al. Visceral artery aneurysms, an experience on 32 cases in a single center: treatment from surgery to multilayer stent. Ann Vasc Surg 2011;25:923-35.

16. Antoniou GA, Schiro A, Smyth JV, et al. Multilayer stent in the treatment of popliteal artery aneurysms. Vasa 2012;41:383-7.

17. Sultan S, Hynes N. Disruptive endovascular technology with Multilayer Flow Modulator stents as a therapeutic option in the management of thoracoabdominal aortic aneurysms. Early results from the global independent MFM registry. J Vasc Endovasc Surg 2012;19:215-28.

18. Sultan S, Hynes N. One-year results of the multilayer flow modulator stent in the management of thoracoabdominal aortic aneurysms and type B dissections. J Endovas Ther 2013;20:366-77.

19. Debing E, Aerden D, Gallala S, et al. Stenting complex aorta aneurysms with the cardiatis multilayer flow modulator: first impressions. Eur J Vasc Endovasc Surg 2014;47:604-8.

20. Sultan S, Kavanagh EP, Bonneau M, et al. Abdominal aortic aneurysm repair using the multilayer flow modulator in porcine animal models. Universal J Med Sci 2015;3:1-10.

21. Sultan S, Kavanagh EP, Hynes $\mathrm{N}$, et al. Evaluation of functionality and biological response of the multilayer flow modulator in porcine animal models. Int Angiol 2016;35:31-9.

22. Sultan S, Kavanagh EP, Bonneau M, et al. Assessment of biocompatibility of the multilayer flow modulator with differing thread designs. J Vasc Med Surg 2015;2:167.

23. Sultan S, Kavanagh EP, Bonneau M, et al. Kinetics of endothelialization of the multilayer flow modulator and single-layer arterial stents. Vascular 2016;24:78-87.

24. Sultan S, Kavanagh EP, Alves A, et al. A pilot study of the intracranial multilayer flow modulator as a new disruptive technology in the treatment of cerebral aneurysms. Med Res Arch 2015;3. doi:10.18103/mra.v0i3.157.

25. Sultan S, Hynes N, Kavanagh EP, et al. How does the multilayer flow modulator work? The science behind the technical innovation $J$ Endovasc Ther 2014;21:814-21.

26. Bui BT, Oliva VL, Leclerc G, et al. Renal artery aneurysm: treatment with percutaneous placement of a stent-graft. Radiology 1995;195:181-2. 
27. Dib M, Sedat J, Raffaelli C, et al. Endovascular treatment of a wide-neck renal artery bifurcation aneurysm. $J$ Vasc Interv Radiol 2003;14:1461-4.

28. Karkos CD, D'Souza SP, Thomson GJ, et al. Renal artery aneurysm: endovascular treatment by coil embolisation with preservation of renal blood flow. Eur J Vasc Endovasc Surg 2000:19:214-16.

29. Tshomba Y, Deleo G, Ferrari S, et al. Renal artery aneurysm: improved renal function after coil embolization. J Endovasc Ther 2002;9:54-8.

30. Robinson WP III, Bafford R, Belkin M, et al. Favorable outcomes with in situ techniques for surgical repair of complex renal artery aneurysms. J Vasc Surg 2011;53:684-91.
31. Lazaris AM, Maheras AN, Vasdekis SN. A multilayer stent in the aorta may not seal the aneurysm, thereby leading to rupture. J Vasc Surg 2012;56:829-31.

32. Antoniou GA, Papas TT, Mazarakis I, et al. Aortic aneurysm rupture following treatment with flow-diverting Multilayer stents. J Endovasc Ther 2013;20:872-4.

33. Lowe $C$, Worthington $A$, Serracino-Inglott $F$, et al. Multi-layer flow-modulating stents for thoraco-abdominal and peri-renal aneurysms: the UK pilot study. Eur J Vasc Endovasc Surg 2016;51:225-31.

34. Sultan S, Hynes N, Sultan M, et al, MFM Collaborators. When not to implant the multilayer flow modulator: lessons learned from application outside the indications for use in patients with thoracoabdominal pathologies. J Endovasc Ther2014;21:96-112. 